\title{
Thiolated Nucleotides for Immobilisation of DNA Oligomers on Gold Surfaces
}

\author{
Benjamin Bornemann, ${ }^{[a]}$ Shou Peng Liu, ${ }^{[b]}$ Artur Erbe ${ }^{[b]}$ Elke Scheer, ${ }^{[b]}$ and Andreas Marx* ${ }^{*[a]}$
}

Thiols have a strong tendency to be chemisorbed to gold surfaces forming self-assembled monolayers. ${ }^{[1,2]}$ The feature of strong $\mathrm{S}-\mathrm{Au}$ bond formation has been exploited in numerous applications including the immobilisation of DNA on gold surfaces for the generation of DNA microarrays or gold electrodes for measuring charge migration mediated by double stranded DNA ${ }^{[3,4]}$ To be immobilised on gold, DNA has to be modified with thiol functionalities. Usually, for this purpose oligonucleotides are equipped with thioalkyl moieties at the $5^{\prime}$ - or $3^{\prime}$-terminus. ${ }^{[5,6]}$ These modifications have been employed in studies dedicated to directly measure electrical transport through DNA molecules. ${ }^{[7,8]}$ However, those transport measurements report contradictory results for the conduction behaviour of DNA. ${ }^{[9]}$ Some of these contradictions could be attributed to influences of the DNA sequence or the environment of the molecules. On the other hand, it is evident that poor overlap of the orbitals of the thiols with the $\pi$-system of the DNA as well as unreliable or unspecific bonding of the thiols to the metal electrodes are most likely playing a major role in causing these contradictions. Along these lines we became interested in the synthesis of oligonucleotides that bear short thioalkyl functions in close proximity to the $\pi$-system of the nucleobase to facilitate electrical transport through dsDNA. ${ }^{[10]}$ Herein we report the synthesis of new thiolated nucleotides, their incorporation in DNA oligonucleotides as well as studies employing fluorescence and atomic-force microscopy for verifying the immobilisation of the thiolated species onto gold substrates.

For the design of the thiol modified nucleosides, future applications for transport measurements are kept in mind. The 5 -position of thymidine was chosen for modification to be in close proximity to the $\pi$-system and to maintain accessibility over the major groove in double strand DNA, thus 1 shown in Scheme 1 bears a thiol-moiety directly attached to the nucleobase that is protected as ether. In another approach thymidine $\mathbf{2}$ is synthesised via the introduction of a thiophene moiety conjugated via an alkyne spacer to the nucleobase.

To commence the synthesis of 1, the protected 5-iodo-2'-deoxyuridine $3^{[11]}$ was first treated with $\mathrm{NaH}$ and after cooling to $-78^{\circ} \mathrm{C}$ with $n \mathrm{BuLi}$ and 1,2-bis(2-(trimethylsilyl)ethyl) disulfane

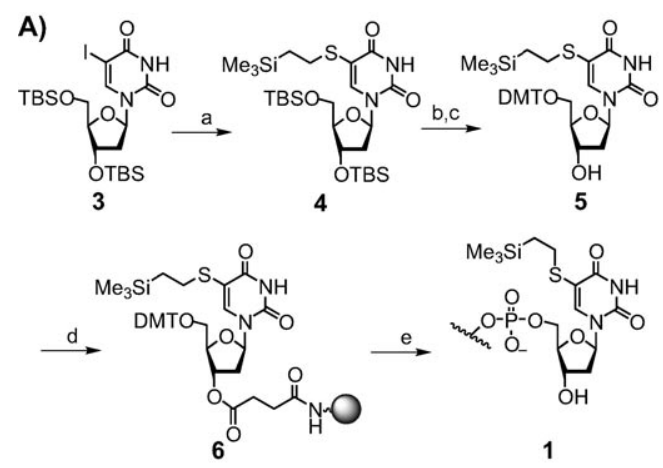

B)
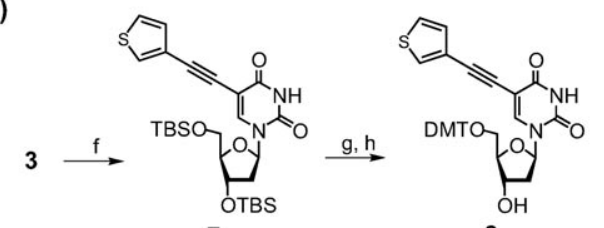

7

8

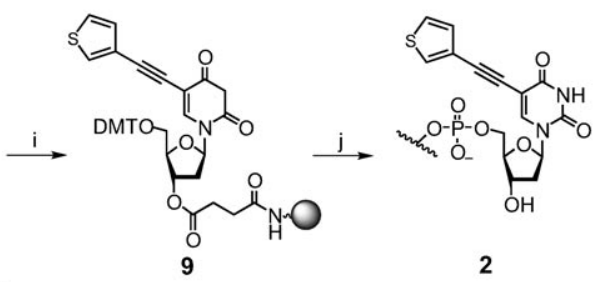

C)

ON1b: 5'-FAM-CGT TGG TCC TGA AGG AGG AT ON2b: 5'-FAM-CGT TGG TCC TGA AGG AGG A1

ON1a: 5'-CGT TGG TCC TGA AGG AGG AT ON2a: 5'-CGT TGG TCC TGA AGG AGG A1 ON3: $\quad$ 5'-CGT TGG TCC TGA AGG AGG A2

Scheme 1. A) Synthesis of 1. a) 1. NaH, THF, $30 \mathrm{~min}, \mathrm{rt} ; 2 . n \mathrm{BuLi},-78^{\circ} \mathrm{C} ; 3$. $\left(\mathrm{Me}_{3} \mathrm{SiC}_{2} \mathrm{H}_{4} \mathrm{~S}\right)_{2}, 12 \mathrm{~h},-78^{\circ} \mathrm{C}$ to rt, $\left.(76 \%) ; \mathrm{b}\right) \mathrm{TBAF}, \mathrm{THF}, 4 \mathrm{~h}, \mathrm{rt},(92 \%)$; c) DMTrCl, DMAP, pyridine, $0{ }^{\circ} \mathrm{C}$ to $\mathrm{rt}, 18 \mathrm{~h},(51 \%)$; d) EDC, DMAP, succinylat ed LCAA CPG, pyridine; then 4 nitrophenol; then piperidine, then acetic an hydride/pyridine/THF (cap A) and 1 methylimidazole/THF (cap B); e) oligonu cleotide synthesis. B) Synthesis of 2. f) 1. $\mathrm{Cul}_{1} \mathrm{Et}_{3} \mathrm{~N}, \mathrm{Pd}\left(\mathrm{PPh}_{3}\right)_{4}, 3$ ethynylthio phene, DMF, $4 \mathrm{~h}(74 \%)$; g) TBAF, THF, 24 h, rt, (96\%); h) DMTrCl, DMAP, pyri dine, $0{ }^{\circ} \mathrm{C}, 6 \mathrm{~h},(76 \%)$; i) EDC, DMAP, succinylated LCAA CPG, pyridine; then 4 nitrophenol; then piperidine, then acetic anhydride/pyridine/THF (cap A) and 1 methylimidazole/THF (cap B); j) oligonucleotide synthesis. C) Synthe sized DNA oligonucleotides. [a] B. Bornemann, Prof. Dr. A. Marx

Department of Chemistry and Konstanz Research School Chemical Biology University of Konstanz, Universitätsstrasse 10, 78457 Konstanz (Germany) Fax: (+49) 7531885140

E mail:andreas.marx@uni konstanz.de

[b] S. P. Liu, Dr. A. Erbe, Prof. Dr. E. Scheer Department of Physics University of Konstanz, Universitätsstrasse 10, 78457 Konstanz (Germany) Supporting information for this article is available on the WWW under http://www.chemphyschem.org or from the author. results in the desired product 4 in good yields (Scheme $1 \mathrm{~A}$ ). Standard protection group manipulations yielded the 5 '-protected nucleoside $\mathbf{5}$ that was subsequently coupled to long chain alkyl amine on a controlled pore glass (LCAA-CPG) support. Interestingly, the (trimethylsilyl)ethyl (TMSE) protection group in $\mathbf{4}$ withstands the treatment with tetrabutylammonium fluoride (TBAF). However since it was reported earlier that thioethers are sufficient for tightly coordinating to gold ${ }^{[12]}$ we 
continued our investigations. In fact, using an entity with protected thiol-functionality turned out to be highly beneficial for handling the DNA samples (vide infra). For the synthesis of thiophene-modified nucleoside 2, 5-iodo-2'-deoxyuridine (3) was treated with 3-ethynylthiophene in a Sonogashira reaction ${ }^{[13]}$ resulting in the desired product 7 (Scheme $1 \mathrm{~B}$ ). Standard protecting group manipulations yielded the 5 -protected nucleoside 8 that was subsequently coupled to the LCAA-CPG support. The modified solid supports 6 and 9 were employed in automated DNA oligonucleotide synthesis to form oligonucleotides that bear the thiolated 2 -deoxyuridine moiety 1 and 2 at the $3^{\prime}$-terminus (Scheme $1 \mathrm{C}$ ). The yields are comparable to the yields obtained for the unmodified reference samples. For subsequent fluorescence microscopy studies (vide infra) a 20 mer oligonucleotide that bears a fluorescent dye (FAM: fluorescein) at the $5^{\prime}$-terminus via a commercially available C6-amino modifier and 1 at the 3'-terminus (ON2b) was synthesised. For atomic-force microscopy (AFM) studies oligonucleotides ON2a and ON3 were synthesised. Mass spectrometric analysis verified the integrity of the oligonucleotides. For reference purposes the same 20 mer oligonucleotides were synthesised that contained an unmodified thymidine moiety at the $3^{\prime}$-terminus instead of the analogues 1 and 2 (ON1a and ON1b).

To verify the propensity of the synthesised oligonucleotides for immobilisation on gold surfaces we performed microscopy studies. Fluorescence microscopy as a sensitive and straightforward tool is used to investigate the immobilisation of modified DNA on a gold substrate. Identical gold patterns were produced by evaporation through a mesh with grid size $10 \mu \mathrm{m} \times 10 \mu \mathrm{m}$ and equal distance. The metal is evaporated with a thickness of $10 \mathrm{~nm}$ onto a glass substrate covered by a $2 \mu \mathrm{m}$ thick polyimide layer. We chose this substrate system because on the one hand, glass as well as polyimide, are transparent enough for transmission optical investigations. On the other hand electron-beam lithography-which is used for fabricating controllable electrode structures for the single-molecule transport experiments-works well on flexible metallic substrates covered by polyimide. ${ }^{[14,15]}$ After breaking the vacuum and removing the mask a drop $(10 \mu \mathrm{L})$ of DNA solution (10 $\mu \mathrm{mol}$ in TBE buffer) is deposited onto the substrate. Incubation continued for two hours before the substrate was rinsed with TBE buffer to remove the unbound molecules. Subsequently, we investigated the immobilisation behaviour of the two species ON1b and ON2b as described by fluorescence microscopy.

Figure 1A, image a shows a picture of immobilised ON2b deposited onto a freshly evaporated gold film. A strong fluorescence signal is observed from the covered metal structures. Except for a few locations we do not see any optical response on the polyimide. Figure $1 \mathrm{~A}$, image $\mathrm{b}$ depicts the result of the equivalent experiment but with the species ON1b, where a very weak optical signal is found. Modified species ON2b binds specifically onto the gold surface, while ON1b physisorbs unspecifically and is consequently rinsed away with the buffer. Control experiments with unprotected thiols show only a very weak fluorescence signal (vide infra). Another possible explana-
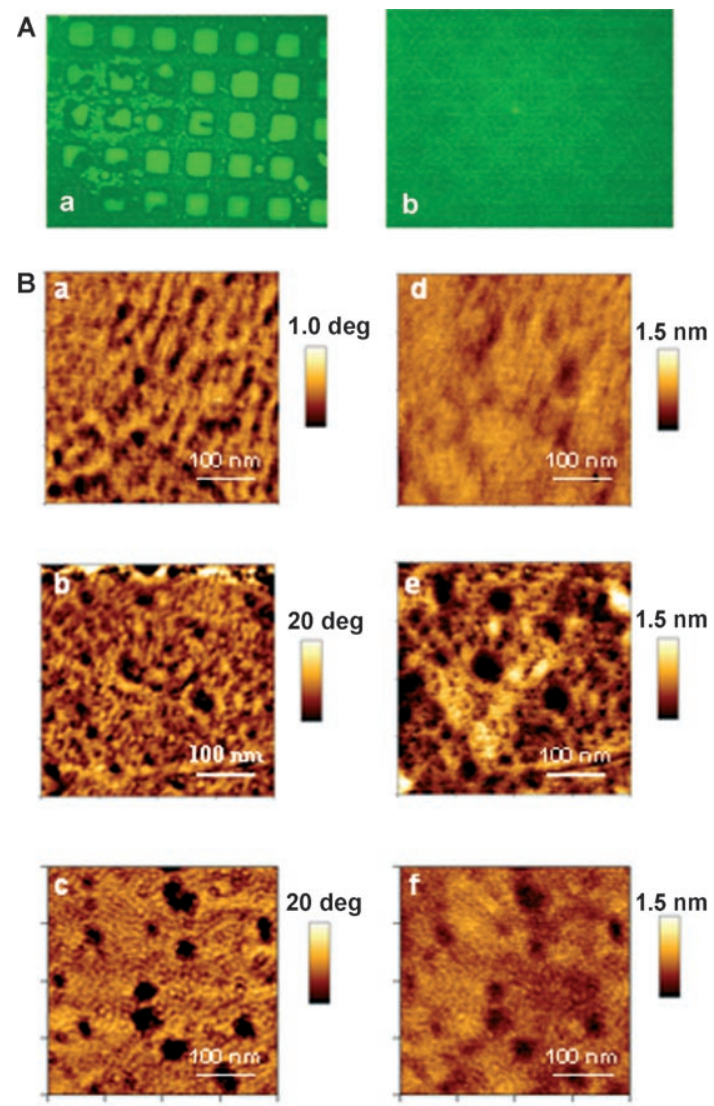

Figure 1. A) Fluorescence microscope images of species ON2b (image a) and ON1b (image b) both containing a fluorescent dye on gold. The absence of contrast in image $b$ reflects the fact that no chemisorption occurred as the molecules were washed away upon rinsing. B) AFM phase contrast images (left) and topography images (right) of species ON1a (a,d), ON2a (b,e) and ON3 $(c, f)$ on gold surfaces. The black spots indicate areas without molecules. The weak phase contrast in image a reflects the absence of molecules. The maximum height variation is $0.7 \mathrm{~nm}$ (image d), $1.4 \mathrm{~nm}$ (image e) and $1.2 \mathrm{~nm}$ (imagef).

tion for the lack of the optical signal in Figure $1 \mathrm{~A}$, image $\mathrm{b}$ would be a quenching of the fluorescence by the close vicinity of the metal layer. However, this possibility is ruled out based on the results of the subsequent AFM studies. We thus conclude that the difference in optical response is indeed due to the different immobilisation strength of the species.

The results of the fluorescence microscopy studies are corroborated by AFM studies. A $200 \mathrm{~nm}$ thick gold layer was evaporated onto a fresh-cleaved mica substrate and the gold surface was glued to a steel substrate using double-sided scotch tape. We then stripped the mica off the gold using tweezers. This procedure results in a relatively smooth gold surface $[0.4 \mathrm{~nm}$ roughness (RMS value) in an area $10 \mu \mathrm{m} \times 10 \mu \mathrm{m}$ ]. Subsequently, a $10 \mu \mathrm{L}$ droplet of the respective oligonucleotide in buffer $(10 \mu \mathrm{mol})$ was deposited. After $1 \mathrm{~h}$ the remaining solution was rinsed with water and the surface dried in a nitrogen stream. The rinsing is performed in pure water in order to avoid artefacts in the AFM images arising from residues of the buffer. The surfaces were investigated with AFM in the dynamic mode. The topographic information with a resolution of ap- 
proximately $0.1 \mathrm{~nm}$ in height was gained, while the phase image gave contrast when material properties like elasticity or viscosity varied over the surface. Although the contrast mechanism is not fully understood, qualitative chemical information can be obtained. ${ }^{[16]}$ For blank gold surfaces the maximum phase shift is smaller than $1 \mathrm{deg}$.

Figure 1B images a-c depict the phase and Figure 1B images $d-f$ the topography images of species ON1a, ON2a and ON3. While the topography image of species ON1a is very smooth and could thus originate from a rather homogeneous molecular film, the phase contrast of Figure $1 \mathrm{Bimage} a$ is very weak. At variance to these findings species ON2a and ON3 show pronounced phase contrast (up to $20 \mathrm{deg}$ ) and topography contrast (up to $1.5 \mathrm{~nm}$ ) at the same positions, while no clear spatial correlation exists between the signals in the phase and height images of ON1a. We interpret the absence of a chemical contrast in Figure $1 \mathrm{~B}$ image a as being due to the fact that the molecules ON1a have been rinsed away, in accordance with the fluorescence microscope result obtained with the species ON1b described above. We conclude that the unthiolated oligomer ON1a does not bind chemically on the gold surface.

The situation is different for molecules of the species ON2a and ON3. Both the height and the phase image show black spots (low height and low phase shift) at the same location. The height difference between the black areas and the higher areas is in the order of $1.4 \mathrm{~nm}$ for ON2a and $1.2 \mathrm{~nm}$ for ON3 and thus smaller than the length of the stretched molecules $(6 \mathrm{~nm})$. We thus conclude that the TMSE protected (ON2a) and thiophene-terminated (ON3) molecules are lying flat or crumbled on the surface. ${ }^{[18]}$ This is a consequence of the small stiffness of ssDNA which hampers brush formation even for high coverages. The phase shift difference is also about a factor of twenty larger than observed for blank gold surfaces and species ON1a. We note that the height images look similar for all species investigated, nevertheless clear differences are observed in the phase signals. The comparison of the results obtained for these three species underlines the importance of investigating more than one contrast mechanism when using atomic force microscopy. All immobilisation investigations, that is, fluorescence spectroscopy as well as AFM have been performed repeatedly without any systematic sample dependence.

We note that equivalent experiments with unprotected thiols showed unreliable bonding, presumably due to faster oxidation of the thiols within a few minutes under the working conditions of the experiments (data not shown). Applying the same immobilisation procedures which were successful for species ON2, produces neither reproducible fluorescence nor AFM signals for molecules with unprotected thiols. This fact may thus explain part of the large variations of transport properties observed in dithiolated aromatic molecules. ${ }^{[18]}$

We summarise that TMSE-protected thiols and thiophenes are suitable endgroups for the immobilisation of organic molecules. This result is important for electronic transport measurements because successful and reproducible charge injection relies on reproducible immobilisation and chemisorption of the molecules under study. We furthermore verified that a fresh and dry gold surface is mandatory for successful bonding of the molecules. With species ON2a, ON2b and ON3, when using substrates which have been exposed to air by more than one hour before the application of the molecular solution, neither clear fluorescence signals nor contrast in the AFM pictures were observed. With ON1 we did not observe successful bonding even when the time between evaporation and deposition was shorter than $10 \mathrm{~min}$. Transport measurements through suspended dsDNA based on species ON2a and ON3 modifications and using the mechanically controllable breakjunction technique $\mathrm{e}^{[14,19]}$ are underway. For this purpose hybridisation of the oligonucleotides with their complementary strand is performed in solution. Since the helical structure gives rise to much larger stiffness the molecules are able to span a gap of nanometer size between two metal electrodes. ${ }^{[20]}$

\section{Experimental Section}

Fluorescence Microscopy: Filters with wavelength of $488 \pm 10 \mathrm{~nm}$ and $520 \pm 10 \mathrm{~nm}$ were used for excitation and emission. The lateral resolution of the imaging system is $130 \mathrm{~nm}$. The size of the images is approximately $87 \mu \mathrm{m} \times 67 \mu \mathrm{m}$.

Further synthesis procedures and measurement (AFM and fluorescence) conditions can be found in the Supporting Information.

\section{Acknowledgements}

We thank H. Li and T. Gisler for assistance with the fluorescence measurements, G. Maret and S. Mecking for use of equipment, and K.-J. Jung for his assistance in the preparation of the manuscript. Funding by the DFG within SPP 1243 is kindly acknowledged.

Keywords: DNA · fluorescence · immobilisation · nucleoside thiol-gold interaction

[1] C. Pale Grosdemange, E. S. Simon, K. L. Prime, G. M. Whitesides, J. Am. Chem. Soc. 1991, 113, 1220.

[2] C. D. Bain, G. M. Whitesides, Angew. Chem. 1989, 101, 522 528; Angew. Chem. Int. Ed. 1989, 28, 506512.

[3] C. A. Mirkin, R. L. Letsinger, R. C. Mucic, J. J. Storhoff, Nature 1996, 382, 607609.

[4] A. P. Alivisatos, K. P. Johnsson, X. Peng, T. E. Wilson, C. J. Loweth, M. P. Bruchez, Jr., P. G. Schultz, Nature 1996, 382, 609611.

[5] B. A. Connolly, P. Rider, Nucleic Acids Res. 1985, 13, 44854502.

[6] N. D. Sinha, R. M. Cook, Nucleic Acids Res. 1988, 16, 26592670.

[7] H. Cohen, C. Nogues, R. Naaman, D. Porath, Proc. Natl. Acad. Sci. USA 2005, 102, 1158911593.

[8] B. Q. Xu, P. M. Zhang, X. L. Li, N. J. Tao, Nano Lett. 2004, 4, 11051108.

[9] D. Porath, G. Cuniberti, R. D. Felice, Top. Curr. Chem. 2004, 237, 183227.

[10] B. Bornemann, A. Marx, Bioorg. Med. Chem. 2006, 14, 62356238.

[11] N. C. Chaudhuri, E. T. Kool, J. Am. Chem. Soc. 1995, 117, 1043410442.

[12] Examples: a) W. M. Pankau, S. Mönninghoff, G. von Kiedrowski, Angew. Chem. 2006, 118, 1923 1926; Angew. Chem. Int. Ed. 2006, 45, 1889 1891 ; b) M. M. Maye, J. Luo, I. S. Lim, L. Han, N. N. Kariuki, D. Rabinovich, T. Liu, C. J. Zhong, J. Am. Chem. Soc. 2003, 125, 99069907.

[13] a) K. Sonogashira, Y. Yohda, N. Hagihara, Tetrahedron Lett. 1975, 16, 4467 4470; b) H. Doucet, J. C. Hierso, Angew. Chem. 2007, 119, 850 888; Angew. Chem. Int. Ed. 2007, 46, 834871. 
[14] J. M. van Ruitenbeek, A. Alvarez, I. Piñeyro, C. Grahmann, P. Joyez, M. H. Devoret, D. Esteve, C. Urbina, Rev. Sci. Instrum. 1996, 67, 108111.

[15] Introducing Molecular Electronics, Lecture Notes in Physics (Eds.: G. Cuni berti, G. Fagas, K. Richter), Springer, Berlin, 2005, pp. 110.

[16] R. García, R. Pérez, Surf. Sci. Rep. 2002, 47, 197301.

[17] A. Y. Kasumov, D. V. Klinov, P. E. Roche, S. Gueron, H. Bouchiat, Appl. Phys. Lett. 2004, 84, 10071009.

[18] H. B. Akkerman, B. de Boer, J. Phys. Condens. Matter 2008, 20, 13001.
[19] N. Kang, A. Erbe, E. Scheer, New J. Phys. 2008, 10, 023030.

[20] S. Roy, H. Vedala, A. Datta Roy, D. H. Kim, M. Doud, K. Mathee, H. K. Shin, N. Shimamoto, V. Prasad, W. Choi, Nano Lett. 2008, 8, 2630.

Received: February 7, 2008

Revised: April 11, 2008

Published online on May 14, 2008 